\title{
Les politiques de communication par le sport au service de la dynamique organisationnelle des entreprises
}

\section{Lilian Pichot}

\section{OpenEdition}

\section{Journals}

Édition électronique

URL : http://journals.openedition.org/communicationorganisation/222

DOI : 10.4000/communicationorganisation.222

ISSN : 1775-3546

\section{Éditeur}

Presses universitaires de Bordeaux

\section{Édition imprimée}

Date de publication : 1 juillet 2007

Pagination : 230-247

ISSN : $1168-5549$

\section{Référence électronique}

Lilian Pichot, «Les politiques de communication par le sport au service de la dynamique organisationnelle des entreprises », Communication et organisation [En ligne], 31 | 2007, mis en ligne le 17 février 2011, consulté le 30 avril 2019. URL : http://journals.openedition.org/

communicationorganisation/222 ; DOI : 10.4000/communicationorganisation.222 
Analyses

\section{Résumé}

Les dynamiques d'entreprise peuvent être associées à la mise en œuvre d'une politique de communication interne et externe liée au sport. Si cette dernière peut accompagner la politique d'entreprise, elle peut aussi soutenir des changements structurels renouvelant les normes de travail habituelles. A partir d'une approche contextuelle et systémique privilégiant la dimension symbolique des échanges, les communications formelles et informelles, notre recherche vise à montrer comment les caractéristiques du fait sportif, les divers usages du sport, en font un " produit communicationnel» légitime pour les entreprises dont l'activité économique est indépendante du sport. Les supports de "communication sportive» définissent et valorisent indissociablement l'identité de l'entreprise et celle des salariés.

Mots-clefs : entreprise - communication sportive - pouvoir symbolique - communautés informelles - identité - micro-culture

\section{Abstract}

The dynamic of a company can be associated with the implementation of a policy of internal and external communication linked with sport. If this last one can follow the politics of company, it can also support structural changes renewing the usual working standards. From a contextual and systemic approach privileging the symbolic dimension of the exchanges, the formal and abstract communications, our research aims to display the characteristics of the sports fact, the different manners of sport, make it a communicationnel " product " justifying for the companies the economic activity which is independent from sport. The supports of " sports communication " define and value indissociably the identity of the company and those of the employees themselves.

Key-words : company - sports communication - symbolic power informal communities - identity - micro-culture

Lilian Pichot est Docteur en sociologie et maître de conférences à l'UFR STAPS de l'université Marc Bloch de Strasbourg, chercheur au sein de l'équipe d'accueil en Sciences du Sport EA 1342. Son domaine de recherche concerne l'analyse des logiques d'action expliquant les politiques de communication par le sport des entreprises non positionnées dans le champ sportif. 


\section{Les politiques de communication par le sport au service de la dynamique organisationnelle des entreprises Lilian Pichot}

pichot@umb.u-strasbg.fr

Les premières recherches dédiées spécifiquement aux politiques de communication par le sport des entreprises «non sportives $»^{1}$ signalent deux orientations principales non exclusives l'une de l'autre. L'une considère le sport comme constituant de l'imaginaire organisationnel et comme outil de cohésion interne dans l'entreprise ${ }^{2}$. L'autre s'attache aux ressorts selon lesquels les entreprises utilisent le sport comme levier de communication externe à effets internes ${ }^{3}$. Si les récentes transformations du management dans l'entreprise (le néomanagement) intronisent le coaching de cadres et le développement personnel typiques d'un usage du sport à des fins de gestion des ressources humaines ${ }^{4}$, la communication des entreprises par le sport demeure un terrain d'investigation et d'analyse encore peu approfondi ne permettant pas d'expliquer son caractère pluriforme.

Fonction dans l'entreprise parmi d'autres (marketing, ressources humaines), la communication est aussi une norme de fonctionnement définissant le travail - «on ne peut pas ne pas communiquer dans l'entreprise »-, voire une idéologie ${ }^{5}$.

Les dynamiques organisationnelles contribuent à la mise en œuvre d'une politique de communication interne et externe adossée au sport ${ }^{6}$. Servant fréquemment à avaliser la politique d'entreprise, la communication peut accompagner des changements structurels renouvelant les normes de travail habituelles? ${ }^{7}$. Des entreprises

\footnotetext{
${ }^{1}$ « Non sportives », c'est-à-dire dont l'activité économique est indépendante du sport (entreprises industrielles, agro-alimentaires, compagnies d'assurance, banques, etc.).

${ }^{2}$ Lire notamment les recherches de F. Burlot mentionnées en bibliographie.

${ }^{3} \mathrm{Cf}$. les travaux de Pichot Lilian cités en bibliographie.

${ }^{4}$ Voir les recherches de B. Barbusse (1997), de H. Mahé de Boislandelle (1997) et de F. Burlot (2001).

${ }^{5}$ Breton Philippe \& Proulx Serge. L'explosion de la communication. La naissance d'une nouvelle idéologie. Paris, La Découverte. 1989.

${ }^{6}$ Pichot Lilian \& Tribou Gary, « Sponsoring sportif : outil de communication externe et interne », in Bouchet P. et Pigeassou C. (dir.), Management du sport : actualités, développements et orientations de la recherche. Montpellier, Ed. AFRAPS, 2006, pp. 391-408.

${ }^{7}$ C'est par exemple le cas en situation de plan social.
} 
Analyses

communiquent par le sport en favorisant une politique intégrée de services aux salariés et leur «développement personnel». Ces dynamiques organisationnelles révèlent des actions collectives non imposées par le sommet hiérarchique mais mobilisant les salariés adhérant à un projet d'actions sportives ${ }^{8}$.

Cette recherche vise à montrer comment les possibles usages du sport en font un "produit communicationnel » pour les entreprises en même temps qu'une ressource pour les salariés. Les supports de communication définissent indissociablement l'identité de l'entreprise et celle des salariés, par la transmission de messages, d'idées, de représentations censés qualifier à la fois l'entreprise et les salariés.

Prenant appui sur le modèle systémique et orchestral de la communication ${ }^{9}$, les « usages communicationnels » du sport relèvent d'un processus social au cœur duquel les différents acteurs sont amenés à mettre en commun, participer, s'impliquer voire à communier. Ces usages sont à resituer dans un contexte environnemental fécondant, par delà l'expression collective de groupes, la nature des échanges et des interactions entre les personnes. Il nous importe d'expliquer dans quelles mesures les salariés participent à « la communication sportive » et fabriquent « de la mise en commun ». En d'autres termes, comment et pourquoi leurs comportements communicationnels à caractère sportif constituent des ensembles significatifs? L'interprétation de la communication d'entreprise conduit à observer le cadre culturel plus ou moins normatif de l'entreprise, les conditions spatiale et temporelle des échanges. L'implication relative des personnes dans les actions sportives est une nécessité de la régulation dans les entreprises, une condition de dynamiques et de changements organisationnels ${ }^{10}$.

Délaissant la nature psychologique des individus et la signification que les salariés attribuent aux actions dans lesquelles ils sont impliqués (perspective théorique constructiviste), nous tenterons d'objectiver le contexte et les règles spécifiques des systèmes dans lesquels ils sont insérés (institutions, groupes, interactions). Notre

\footnotetext{
${ }^{8}$ Par exemple, adhérer à l'association sportive, participer aux raids interentreprises, aux conférences de personnalités sportives sur le management des équipes, suivre des sessions de formations intégrant des jeux de rôles sportifs, etc.

${ }^{9}$ Winkin Yves et alii. La nouvelle communication. Paris, Seuil, coll. « Points ». 1981.

${ }^{10}$ Nous entendons par dynamiques organisationnelles, des mobilisations collectives des salariés des entreprises sur le mode du volontariat, la création de liaisons et d'échanges sociaux entre les services et entre leurs membres et la création des conditions institutionnelles et informelles d'un nouvel « être ensemble».
} 
recherche vise à déconstruire les modalités de formation de cette politique et les ressorts institutionnels - endogènes et exogènes - et personnels à l'origine $\mathrm{du}$ processus. D'inspiration systémique, l'analyse se focalisera sur les modalités collectives de la participation des acteurs, les conditions contextuelles des initiatives proposées en matière de communication autour du sport ${ }^{11}$ et de la mobilisation.

La politique communicationnelle peut ainsi dévoiler :

- une stratégie visant un objectif en fonction des ressources possédées, le sport étant instrumentalisé sous différentes modalités,

- des identités et des histoires individuelles et collectives,

- une micro-culture formée et reconstituée autour de codes communs, pouvant s'inscrire dans un cadre stratégique et refléter aussi les identités.

Le sport et l'entreprise : une association raisonnée et prescrite dans le cadre d'une stratégie communicationnelle

Fait social, le sport est devenu un produit « omnibus » au regard de la diversification des activités sportives corrélative de la diversité sociologique des pratiquants. Il intéresse de près ou de loin, pratiquants ou non, une majeure partie de la population française ${ }^{12}$. Par conséquent, les entreprises affirment sereinement vouloir accorder plus de place au sport dans l'organisation de travail, c'est-à-dire à un centre d'intérêt de masse.

\section{Le pouvoir symbolique du sport ou son usage légitime dans l'entreprise}

Les discours performatifs délivrés dans l'espace marchand s'appuient sur les dimensions non marchandes et désintéressées du sport et sur ses valeurs (fair play, loyauté, esprit d'équipe, etc.). La communication autour de pratiques sportives, éloignées des enjeux principaux de l'organisation, constitue une garantie de la persuasion de ces discours. L'activité communicationnelle autour du sport impose

\footnotetext{
${ }^{11} \mathrm{cf}$. annexe méthodologique en fin d'article.

${ }^{12}$ En référence à l'enquête réalisée par l'INSEP et le Ministère des Sports en 2000 cf. Mignon Patrick, G., Truchot Guy. Les pratiques sportives des Français. Paris, Ministère des sports/INSEP. 2002, les taux de pratique sportive en 2000 varient entre $66 \%$ et $83 \%$ selon les catégories socioprofessionnelles. En 1994, 90\% des Français achètent des biens sportifs (cf. Pouquet Laurent. Le comportement des consommateurs d'articles de sport. Paris, Credoc. 1994).
} 
Analyses

de relever le caractère fonctionnel des mises en situation sportives. Ces dernières sont censées susciter des inférences, des transferts cognitifs et affectifs par la ressemblance des situations vécues. Selon le mimétisme entre les situations de travail et les activités sportives, l'engagement repose sur la croyance que les émotions vécues garantissent le transfert.

«Le souvenir positif d'avoir évolué en équipe est une source de progression au travail (...) Même si nous n'avons pas de preuve tangible, nous n'avons rien mesuré, nous sommes intimement convaincus que ces mises en situation sportives profitent à chacun des participants, en termes de motivation, d'intérêt au travail, de résolution de problème à partir de solutions collectives $»^{13}$. " $\mathrm{C}^{\prime}$ est certain qu'il existe un parallèle entre la réussite commerciale et la réussite sportive, entre une équipe de sportifs et une équipe de vendeurs (...) la préparation de la visite d'un client s'apparente à la préparation d'une rencontre sportive en termes de concentration $»^{14}$.

Le "faire " n'est pas la seule dimension de l'apprentissage de la culture entrepreneuriale. L'activité communicationnelle repose aussi sur la symbolique des mots, des discours managériaux. Outre les symboles communément associés au fait sportif et en particulier à «l'esprit sportif»-1'aventure, l'esprit d'équipe et la solidarité, la progression et l'apprentissage, la réussite individuelle et la performance-, le versant interprétatif permet à chaque salarié d'y déceler des identifications et de justifier ses attitudes et ses comportements. Les symboliques sportives se sont forgées sur la formation des héros sportifs qui incarnent des modèles d'excellence corporelle, des figures de la réussite individuelle et de la communauté d'appartenance ${ }^{15}$.

De plus, les mises en situation et en présence physique par le sport forment des réseaux sportifs qui diffusent dans les services des informations relatives à la vie de l'entreprise. Moyen d'épanouissement pour le personnel, les activités sportives d'entreprise créent des réseaux internes qui augmentent l'interactivité relationnelle, la fréquence de circulation des informations ${ }^{16}$. Le cercle

\footnotetext{
${ }^{13}$ Extrait d'un entretien - Directeur de la communication France Wurth.

${ }^{14}$ Extrait d'un entretien - Directeur marketing France Wurth.

15 Duret Pascal. L'héroïsme sportif. Paris, PUF. 1993. Defrance Jacques. Sociologie du sport. Paris, La Découverte, coll. « Repères ». 1995.

${ }^{16}$ Burlot Fabrice. «Le sport en entreprise : un vecteur de communication interne. » in Revue STAPS, revue internationale des sciences du sport et de l'éducation physique, Grenoble, Ed. AFRAPS, 53, 2000. 65-78.
} 
relationnel de chaque salarié est étendu par les groupements sportifs et touche des services indépendants du champ sportif. Se jouent là les identités des salariés et de l'entreprise.

Si les rencontres sportives entre différents unités de l'entreprise marquent une pause dans la chronologie entrepreneuriale du temps, dans le cycle de l'urgence, les mises en situation rappellent par analogie la contrainte nécessaire de la gestion du temps. Néanmoins, ces espaces temps secondaires et interstitiels dans l'entreprise sont des espaces de liberté pleinement vécus selon le point de vue des managers, et qui contribuent à l'expression de soi, l'engagement personnel comme étape intermédiaire de l'estime de soi.

Si les politiques de communication observées n'énoncent pas leurs objectifs et ne disent pas leur nom, elles persuadent par la nature même des actions proposées. Leur légitimité se conquiert sur la satisfaction des passions des salariés et des clients, par la combinatoire des actes et de la parole.

« On a des collaborateurs qui participent aux associations sportives, les uns jouent aux boules, au foot, aux quilles, les autres participent aux compétitions de ski à l'intérieur du réseau, d'autres vont faire du vélo, etc. (...) On est une entreprise citoyenne, on a des collaborateurs et des clients et un établissement financier doit normalement pouvoir partager leurs passions sportives $»^{17}$.

Le recours au sport pour communiquer dans des situations de transformations structurelles et de conjonctures de changement

Des entreprises accompagnent bien souvent les étapes de leur développement par une nouvelle identité textuelle et visuelle: le logotype de l'enseigne est "modernisé » et symbolise ses activités. Au tournant des années 1990, la transformation du statut juridique de la Caisse d'Epargne (passage du statut d'établissement de crédit à but non lucratif à celui de coopérative) engage la banque dans l'économie de marché concurrentielle. Ce changement l'a conduit à redéfinir son identité visuelle et redessiner son logo sous une griffe plus "hightech », l'écureuil étant stylisé sur un carré, "signe de force » évoquant la technicité, la compétence et l'entrée dans la compétition internationale. Cette rupture marquait alors le lancement d'une nouvelle communication institutionnelle ciblée sur le sport et le choix

\footnotetext{
${ }^{17}$ Extrait d'un entretien - Directeur du Développement d'un groupe bancaire français.
} 
Analyses

de la course à pied comme axe national de sponsoring décliné au sein des directions régionales. ${ }^{18}$

Dans une phase d'ouverture des marchés à la libre concurrence, l'Electricité de Strasbourg, filiale de l'Electricité de France, distributeur historique d'électricité, doit aussi se positionner en tant que fournisseur d'électricité en Alsace. "Pour une entreprise qui se transforme, la communication institutionnelle est primordiale. Après la création d'un nouveau logo, notre positionnement est de nous faire connaître des professionnels et des particuliers, nous avons 450000 clients (...) la culture sportive en interne, elle n'est plus à démontrer, on a 1500 adhérents à l'association sportive et le sport s'est imposé naturellement comme moyen de communication. Un an avant l'ouverture des marchés, on avait besoin de notoriété régionale et on s'est rendu compte qu'un autre media possible, c'était le sponsoring sportif $»^{19}$. L'Electricité de Strasbourg décide alors d'être le partenaire principal à domicile du club de football professionnel local et affiche son nom sur la tenue des joueurs en vue d'obtenir une forte visibilité. En période de plans sociaux en 2004, l'entreprise Steelcase ${ }^{20}$ accompagne la restructuration de ses sites de production et la réduction des effectifs salariés par une politique de communication institutionnelle à fort ancrage régional en devenant également partenaire du club sportif phare de l'élite alsacienne. Cet engagement réside dans la volonté «d'établir la marque Steelcase beaucoup plus fortement qu'elle ne l'est aujourd'hui. Faire en sorte que les cibles institutionnelles régionales aient une image réelle de l'entreprise, d'un acteur économique fort de la région. Pour la survie de l'entreprise, il faut avoir une relation forte avec la communauté urbaine de Strasbourg. C'est très tactique. Le sport concerne uniquement la région. Nous n'avons pas d'ambition de rayonner au-delà de la région via ce sponsoring $»^{21}$. Ce partenariat a aussi une fonction de soutien structurel, en particulier de communication commerciale au sein du

\footnotetext{
${ }^{18}$ Pichot Lilian. La construction d'une politique de communication de l'entreprise. Stratégies de partenariat et développement des organisations sportives dans les secteurs public et privé. Villeneuve d'Ascq, Presses Universitaires du Septentrion. 1999.

${ }^{19}$ Extrait d'un entretien réalisé avec le responsable Communication interne et institutionnelle de l'Electricité de Strasbourg.

20 L'entreprise Steelcase est spécialisée dans la fabrication, la distribution de mobiliers de bureaux, et l'aménagement des espaces tertiaires. Le siège de Steelcase international qui englobe l'ensemble des pays en dehors de l'Amérique du Nord se trouve à Strasbourg.

${ }^{21}$ Extrait d'un entretien réalisé avec la directrice de la communication institutionnelle et interne de Steelcase.
} 
réseau régional de concessionnaires: "On permet à nos concessionnaires d'inviter une cinquantaine de clients pour faire des relations publiques et créer un trafic d'affaires $»^{22}$. Parallèlement, la communication externe est un levier d'opérations de «séduction » en interne. L'ensemble des 1400 collaborateurs de la région peuvent bénéficier de places à tarif réduit et l'entreprise organise des opérations portes ouvertes avec un stand dédié au partenariat avec le club $^{23}$. Globalement, les entreprises qui s'engagent dans une politique de communication institutionnelle et commerciale par le vecteur sportif cherchent à établir des liaisons durables entre les collaborateurs. Elles visent à pallier l'éclatement géographique des établissements de travail et à faire se rencontrer les différents acteurs intermédiaires : agents généraux, concessionnaires, etc. Cette stratégie de communication permet de fusionner les sous-cultures de groupes professionnels, de lisser les différences de culture de travail entre les sites de production occupés par les ouvriers qualifiés et agents de maîtrise et les unités décisionnelles composées quasi-exclusivement de cadres. Dans un contexte d'érosion des discours performatifs des dirigeants face aux salariés incrédules, les espaces d'expressions corporelles et d'actions constituent une alternative intéressante. L'ingénierie symbolique ${ }^{24}$ se déplace pour être en prise sur des objets «neutres », périphériques à l'activité économique fondatrice de l'entreprise. En d'autres termes, l'ingénierie symbolique est d'autant plus efficace qu'elle est construite dans des espaces à la fois extérieurs et connexes à l'entreprise, concernant pour partie les loisirs des salariés.

\section{Des histoires individuelles et collectives à la formation de micro-cultures sportives}

Le contexte culturel de l'entreprise est étroitement lié aux cultures individuelles pour partie importées d'autres espaces sociaux. En ce sens et en suivant Umberto $\mathrm{Eco}^{25}$, la culture doit être étudiée comme un "phénomène de communication fondé sur des systèmes de signification». Ces derniers sont à décrypter par l'analyse des

${ }^{22}$ Ibid.

${ }^{23}$ A cette occasion, les salariés pouvaient rencontrer les joueurs professionnels et le personnel d'encadrement de l'équipe et bénéficiaient de tarifs réduits pour acheter des biens à l'effigie de l'équipe.

${ }^{24}$ Villette Michel. L'homme qui croyait au management. Paris, Seuil. 1988 ; Floris Bernard. La communication managériale. Grenoble, PUG. 1996.

${ }^{25}$ Eco Umberto. A Theory of Semiotics. Bloomington. Indiana University Press (traduction française aux Editions Complexe, Bruxelles). 1976. 
Analyses

conditions d'expression des salariés à travers les pratiques et les thématiques sportives.

\section{Faire, faire dire pour faire faire, pour convaincre et séduire}

La politique de communication vise à toucher l'essence même de «l'être social» et à satisfaire des dispositions et des compétences individuelles acquises dans l'espace sportif, puis importées et exprimées au travail. Les entreprises proposent ainsi des situations pratiques collectives qui permettent de valoriser les salariés et favorisent leur reconnaissance et leur estime de soi. Délestés temporairement de leurs fonction et statut professionnels, ces derniers mobilisent leurs attributs personnels (dispositions physiques, facultés techniques et tactiques, qualités de management, d'encadrement des équipiers notamment). En ce sens, les dynamiques organisationnelles sont subordonnées à la multiplicité des reconnaissances individuelles attestées dans l'espace professionnel et pourtant acquises dans des sphères d'activités extérieures, dans les milieux sportifs.

Par quels moyens la « communication sportive» contribue non moins à définir un nouvel espace « d'être et de vivre ensemble», en quelque sorte un monde communautaire ? Les formes d'action participatives trouvent un terrain privilégié dans les activités sportives pour produire $\mathrm{du}$ lien social entre les salariés. Celles-ci offrent un espace d'expressions physiques et verbales, de communication pratique générant des mises en action, des échanges d'informations, sans solliciter des explications savantes. Les entreprises ont parfois recours à des communicateurs extérieurs, agents de liaison et de médiation entre la direction et les salariés ${ }^{26}$. Les objectifs recherchés sont d'autant plus perçus à la fois comme honnêtes et fragiles - les salariés interagissent avec le communicateur neutre et impartial, même si ce dernier subit de manière variable le poids de l'organisation qui l'emploie et qui peut restreindre son champ d'action - et les situations vécues sans contrôle que le communicateur est étranger à la vie de l'entreprise.

Une des conditions de la reconnaissance repose sur le principe de solidarité et le sentiment partagé d'avoir conscience de contribuer à une œuvre collective. C'est l'une des raisons pour lesquelles l'instauration au quotidien de situations collectives vécues physiquement et plutôt intensément, à la marge de l'activité de travail en d'autres lieux («mises au vert» pour reprendre le jargon employé

${ }^{26}$ Schaeffer Pierre. Les machines à communiquer. Paris, Seuil. 1970. 
par les équipes sportives), facilite la construction d'une unité de conceptions de l'entreprise et de la vie quotidienne en son sein. En effet, les participants vivant les mêmes expériences, acquièrent des propriétés semblables, font partie d'un cercle qu'ils ont activement constitué et sont sensibilisés aux symboles caractérisant ces «aventures» collectives. A l'instar des analyses de Gerbner ${ }^{27}$ à propos de la fonction de socialisation de la télévision, des espaces communautaires dans l'entreprise, en l'occurrence des lieux sportifs, consistent à transmettre et à stabiliser des modèles sociaux, d'excellence comportementale. Les actions exploitant le vecteur sportif socialisent des rôles standardisés, par exemple le leader, les suiveurs dans un groupe de sportifs.

Afin de convaincre les salariés de la possibilité de s'accomplir au travail, les actions initiées en matière de formation du personnel exploitant des moyens comportementaux, non verbaux, comme le support sportif, privilégient le passage à l'acte, « l'agir » corporel dans les relations interpersonnelles. Sans montrer le caractère intentionnel de l'action, les supports pédagogiques et expressifs à caractère sportif peuvent avoir pour effet conditionnel de modifier les comportements, de les renforcer ou de générer des actes nouveaux. En cet état d'imprévisibilité des réponses, ces situations contenant une part d'incertitude sur les effets attendus attestent de l'honnêteté de l'entreprise de participer au «développement personnel» de ses salariés.

Ce contexte construit par l'entreprise qui enjoint chaque collaborateur à devenir l'entrepreneur de sa propre existence et un acteur responsable de sa destinée relève d'une communication de l'ingénierie organisationnelle de l'implication. Les situations de communication tendent à rendre acceptable et prédominant l'agir «qui se gouverne par rapport à ses propres fins $\gg{ }^{28}$. Les discours suggérant le réenchantement du lieu de travail se réalisent en fait aux marges de l'activité économique principale, les pratiques articulant l'individualité et le collectif, l'un et l'autre étant mutuellement subordonnés.

On comprend la valeur que prennent les activités sportives lorsqu'elles sont le résultat d'une démarche de projet, d'une définition de règles qui impliquent la préoccupation d'afficher et d'affirmer une

${ }^{27}$ Gerbner George et al., " Charting the mainstream : television's contributions to political orientations », Journal of Communication, 32, Oxford, Oxford University Press, 1982, pp. 100-127.

${ }^{28}$ Habermas Jürgen. Théorie de l'Agir communicationnel. Paris, Fayard. 1987. 
finalité, un but à atteindre. Les communications sportives encouragent une dynamique combinatoire de différentes rationalités des "acteurs projets » : l'individuel et le collectif, le site de travail et $\langle\text { l'ailleurs }\rangle^{29}$, la gestion des dispositions physiques et morales. Elles cristallisent les imaginaires sociaux et professionnels dans des rapports de proximité entre les collaborateurs et leurs services, bref construisent des liens collectifs symboliques mais réels, de solidarité, d'entraide et d'entente, de réussite et d'excellence individuelle et collective. S'opère alors un glissement de fonction selon lequel la communication entrepreneuriale par le sport entre dans une configuration de gestion humaine des individus.

Si l'entreprise parvient à reconnaître dans la sphère du travail des dispositions personnelles des salariés construites indépendamment de l'univers professionnel, alors elle réussit son opération subreptice de séduction : elle les flatte et les courtise à leur insu.

\section{Des logiques communicationnelles «sportives» facilitant les communautés informelles et les échanges interpersonnels}

Dans quelles conditions le sport offre-t-il des espaces d'autonomie et d'action dans le cadre du travail, connexes à l'activité principale de l'entreprise, potentiellement assimilés à des espaces de coordination, de collaboration, d'échanges entre les salariés ? Les passions sportives gouvernent les modes d'échanges entre les salariés au travail. Si les protagonistes ont conscience des actions auxquelles ils participent et qui ont pour effet induit de satisfaire les dirigeants, ils y trouvent un intérêt personnel extra-professionnel, à valeur biographique. Ainsi ils peuvent s'engager sans avoir une conscience claire des motifs pour lesquels ils agissent. Ces motifs ont des liens ténus avec leur histoire personnelle et collective, notamment leur affiliation aux groupes de sportifs.

C'est une sociologie des pratiques culturelles (dont sportives), qui permet de comprendre l'engagement des personnes dans des sphères d'activité où l'affectivité et les imaginaires tiennent une place centrale dans la mobilisation. Dans la filiation des théoriciens de la culture ${ }^{30}$ travaillant sur des sociétés complexes, les différents services d'une

\footnotetext{
${ }^{29}$ Comme par exemple la participation d'une équipe de salariés à un raid d'aventures sportives dans une autre région ou la pratique du team building dans des cadres spatiaux inhabituels sur les thèmes de la gestion de projet ou du management d'équipe.

${ }^{30}$ Notamment Hall S., «Culture, the media and the ideological effect », in Curran J., Gurevitch N. (eds.), Mass Communication and Society. Adward Arnold, 1977; Williams R., Culture and society, Penguin. 1961.
} 
entreprise ou encore les membres d'un même département peuvent être reliés par une "culture » in situ ce faisant, c'est-à-dire par la production des systèmes de symboles et des messages, notamment ceux qu'offrent les activités physiques et sportives.

Le contexte social et institutionnel pèse sur les communications informelles et la dimension symbolique des relations au sein d'une organisation (prestiges, rites, etc.). L'enjeu vise la construction d'un ensemble commun de référents identitaires et culturels et plus exactement la (re-)création d'une micro-culture dans l'entreprise. Des engagements personnels peuvent s'opérer sous la forme d'actions collectives organisées par un ordre indigène, sacralisées en quelque sorte par leurs auteurs parce qu'elles touchent leur intimité biographique, leur propre vécu sportif. Affranchi de l'ordre entrepreneurial, ce type d'organisation autonome crée une communauté de liens qui pacifient le rapport à autrui. Indirectement, les espaces d'actions sportives relèguent un ordre hiérarchique vertical et atténuent la force de la relation contractuelle du salarié à son entreprise. Des actions de groupements collectifs non institutionnalisés (des salariés sportifs auto-organisés qui s'adonnent à des jeux collectifs sur le lieu de travail mais hors du temps de travail) ont pour effet de pacifier les rapports des salariés à leur entreprise dans des circonstances de restructuration, de pression commerciale liée à des objectifs de compétitivité. Et ce d'autant plus que les relations de coopération au travail s'appuient sur des dispositions durables des personnes ${ }^{31}$ et s'enracinent sur des tranches de vie aux caractéristiques communes. Cette réalité produit une forme de sociabilité propre, un esprit collectif et des valeurs morales partagées. Un groupement de sportifs se rassemble autour d'un intérêt commun pour la pratique, pour son caractère ludique et/ou compétitif et nourrit la vie du collectif par un ensemble de rituels et d'objectifs sportifs et extra-sportifs ${ }^{32}$.

Les modes de coordination et de coopération entre les acteurs sont construits autour de référentiels d'action qui placent la notion de compétence au centre du processus (être performant, savoir prendre les bonnes décisions, savoir être, etc.). L'agir ensemble est orienté par

\footnotetext{
31 Gheorghiu Mihaï Dinu et Moatty Frédéric. «Groupes sociaux et enjeux de la coopération au travail dans l'industrie. » in : Réseaux, Paris, Editions Hermès, vol. 2324, 2005, pp. 91-122.

${ }^{32} \mathrm{Si}$ faire partie d'une équipe sportive constituée par le biais de l'entreprise, c'est pouvoir pratiquer régulièrement avec des collègues de travail, c'est aussi participer à la vie sociale du groupement, à ses soirées, à ses loisirs et côtoyer des personnes devenues des amis.
} 
Analyses

les normes de compétences, par la valorisation du sens pratique. Les formations de mises en situations sportives participent à la recherche de congruence entre les projets individuels de performance, d'intégration à l'intérieur d'une équipe de travail et les projets collectifs de l'organisation, entre des rationalités a priori non convergentes $^{33}$. A l'instar des projets d'équipes sportives, les formes d'organisation participatives et coopératives contribuent à l'échange verbal d'informations, à l'interaction physique et fécondent une démarche d'intercompréhension en vue de l'entente entre les individus et du succès pratique ${ }^{34}$. Dans des situations de communication et des environnements incertains et/ou méconnus, la résolution de problèmes - affronter un adversaire ou un obstacle - nécessite de mobiliser des compétences cognitives, physiques, techniques et motrices et imprime un langage indigène. Des compétences secondaires dans l'entreprise un atout sportif, une habileté technique particulière - deviennent prioritaires et sont valorisées. Les valeurs d'esprit d'équipe, de sens des responsabilités, de prise d'initiative, le goût de l'effort et de la performance dont l'entreprise se réclame pour fabriquer sa «culture d'entreprise » sont patentes au cours de mises en situation réglées et codifiées.

En considérant l'espace de travail comme position d'appréciation, le caractère désintéressé rend essentielle et "authentique» l'action indissociablement individuelle et collective développée par les pratiques sportives.

Pour faire sienne une manière d'appréhender autrui, son espace de travail, pour intégrer des perceptions positives de l'entreprise et des collègues, la reconnaissance de valeurs morales exige de construire des mondes ou des «communautés» qui octroient des marges d'initiative. Dans ce type de sociabilité ouverte, l'éthique de conviction fonctionne comme propriété d'accès et de maintien dans le groupe. Ces microsociétés mettent en évidence l'existence de systèmes de relations et de connexions entre les acteurs ${ }^{35}$ qui permettent de transcender une condition de salarié quelque peu figée. Le «monde» formé féconde l'implication délibérée, objective et subjective des salariés. C'est une condition nécessaire à la formation

\footnotetext{
${ }^{33}$ Chauvin Didier. « La formation comme vecteur de communication. », pp. 115-132, in: Delcambre P. (dir.), Communications organisationnelles. Objets, pratiques, dispositifs. Rennes, Presses Universitaires de Rennes. 2000.

${ }^{34}$ Habermas Jürgen, Théorie de l'Agir communicationnel. op. cit.

35 Borzeix Anni, Bouvier Alban, Pharo Patrick (dir). Sociologie et connaissances. Nouvelles approches cognitives. Paris, CNRS Editions. 1998.
} 
de solidarités « internes » et de sociabilités qui tirent leur quintessence dans le fait de vivre et d'éprouver individuellement et collectivement des moments singuliers, en marge de l'étau entrepreneurial.

\section{Conclusion}

Fait social partie prenante des imaginaires contemporains légitimés du fait de la diversification de la culture de masse ${ }^{36}$, le sport est l'objet permanent d'appropriation par l'individu et les organisations. Il contribue à l'imprégnation d'un système cohérent de pratiques et de signes qui qualifie l'entreprise et lui confère un sens par une symbolique traduisant les valeurs qu'elle diffuse. Les relations entre les personnes, entre les services, par l'entremise du sport, accréditent la structure organisationnelle et définissent une configuration qui forge l'identité de l'entreprise et instaure de nouveaux rapports au travail. Instrumentalisé, le sport correspond à une production collective dans un cadre de travail structuré et hiérarchisé et où les acteurs sont impliqués indépendamment de leur statut. Il permet de relier physiquement voire «moralement» les salariés entre eux, de les satisfaire et d'aiguiser leur sentiment d'appartenance. La communication par le sport est un révélateur de la structure et de la situation de l'entreprise. Outre son caractère fonctionnel, la communication d'entreprise est un fait de structure, un constituant de l'identité entrepreneuriale. L'instrumentalisation du sport, comprise et admise par les salariés, n'est pas incompatible avec leurs préoccupations personnelles. Parce qu'elle ne correspond pas à une série d'injonctions, la communication par le sport leur offre des espaces d'autonomie inédits, plus ou moins contraints mais réels, qui définissent des nouveaux modes de relation et de mobilisation au travail. Productrices d'actes et de symboles qui s'interprètent et assurent une transmission, les activités sportives vécues ou les thématiques sportives traitées par des conférenciers cristallisent des rapports sociaux construits sur des relations extra-professionnelles. Dans cet ensemble communicationnel, un socle de références communes, de savoir-être, un contexte d'expressions atypiques déconnectées de la tradition professionnelle, un cadre de pensée suggérant des valeurs et des règles, créent les conditions de possibilité de micro-cultures groupales, de cohésion et de régulation autonome de

${ }^{36}$ Ohl Fabien, «Goût et culture de masse : l'exemple du sport. » in : Sociologie et sociétés, Montréal, Les Presses de l'université de Montréal, vol. XXXVI-1, 2004, 209-228. 
Analyses

relations. Contribuant à diffuser l'image voulue de l'entreprise, la communication par le sport cimente les relations intra et inter groupes, elle offre une raison d'être aux salariés et remplit indirectement une fonction d'intégration et d'adaptation à un collectif, du groupement informel aux entités plus vastes. Finalement, les mouvements d'entreprise structurés autour du sport par l'ensemble des acteurs sociaux engagés dans la communication d'entreprise sont aussi structurants. Ils participent à la dynamique organisationnelle, au renouvellement des pratiques et des normes, à la condition néanmoins qu'une partie des acteurs sociaux possède un intérêt minimum pour le sport.

\section{Bibliographie}

Barbusse B., Sport et entreprise : des apports réciproques en matière de gestion des ressources humaines. Thèse de doctorat STAPS, Université René Descartes. Paris. 1997.

Bernoux P., Sociologie du changement dans les entreprises et dans les organisations. Paris, Editions du Seuil. 2004.

Boltanski L., Chiapello E. Le nouvel esprit du capitalisme. Paris, Gallimard. 1999.

Borzeix A., Bouvier A., Pharo, P. (dir). Sociologie et connaissances. Nouvelles approches cognitives. Paris, CNRS Editions. 1998.

Breton P., Proulx S., L'explosion de la communication. La naissance d'une nouvelle idéologie. Paris, La Découverte. 1989.

Burlot $\mathbf{F}$., Le sport en entreprise : un outil de cohésion interne. Enjeux identitaires et mécanismes idéologiques, Thèse de doctorat STAPS non publiée, laboratoire CRCS de la division STAPS d'Orsay, Université de Paris XI Orsay, 1997.

Burlot F. «Le sport en entreprise : un vecteur de communication interne », in : Revue STAPS, revue internationale des sciences du sport et de l'éducation physique, 53, Grenoble, Ed. AFRAPS, 2000. pp. 6578.

Burlot F., «L'intégration du sport dans une politique de gestion des ressources humaines. Le cas d'Apple». In Desbordes M. (coord.), Stratégie des Entreprises dans le Sport. Acteurs et Management. Paris, Economica. 2001. pp. 181-195.

Burlot F. et Pichot L. "L'événement sportif et la cohésion des salariés en entreprise », in Desbordes M. (dir.), Stratégie des entreprises dans le sport. Paris, Economica. 2004. pp. 221-248. 
Les politiques de communication par le sport...

Burlot F., «Sport et identité d'entreprise. Vers une modification de l'imaginaire organisationnel ", in : Revue STAPS, revue internationale des sciences du sport et de l'éducation physique, 69, De Boeck université. 2005, pp. 73-92.

Defrance J., Sociologie $d u$ sport. Paris. La Découverte, coll. « Repères ». 1995.

Delcambre P. (dir.). Communications organisationnelles. Objets, pratiques, dispositifs. Rennes, Presses universitaires de Rennes. 2000.

Duret P., L'Héroüsme sportif. Paris, PUF. 1993.

Eco U., A Theory of Semiotics. Bloomington. Indiana University Press (traduction française aux Editions Complexe, Bruxelles). 1976.

Floris B., La communication managériale. Grenoble, Presses Universitaires de Grenoble. 1996.

Gerbner G., Gross L., Morgan M., Signorelli N., "Charting the mainstream: television's contributions to political orientations ", Journal of Communication, 32, Oxford, Oxford University Press, 1982, pp. 100-127.

Gheorghiu M. et Moatty F., "Groupes sociaux et enjeux de la coopération au travail dans l'industrie.» in : Réseaux, vol. 23-24, Paris, Editions Hermès, 2005, pp. 91-122.

Habermas J., Théorie de l'Agir communicationnel. Paris, Fayard. 1987.

Hall S., "Culture, the media and the ideological effect », in Curran J., Gurevitch N. (eds.), Mass Communication and Society. Adward Arnold, 1977.

Lipiansky E.-M., Identité et communication. Paris, PUF. 1992.

Mahe de Boislandelle $\mathbf{H}$., «De l'organisation efficace au management des ressources humaines ", in : Pigeassou C. et Garrabos C. (dir.), Management des organisations de services sportifs. Paris, PUF, 1997, pp. 154-177.

Mignon P., Truchot G., Les pratiques sportives des Français. Paris, Ministère des Sports et INSEP. 2002.

Mucchielli A., Guivarch J., Nouvelles méthodes d'étude des communications. Paris, Armand Colin. 1998.

Mucchielli A., Corbalan J.-A., Ferrandez V., Théorie des processus de la communication. Paris, Armand Colin. 1998.

Ohl F., "Goût et culture de masse : l'exemple du sport.» in : Sociologie et sociétés, vol. XXXVI-1, Montréal, Les Presses de l'université de Montréal, 2004, pp. 209-228. 
Analyses

Pichot L., La Construction d'une politique de communication de l'entreprise. Stratégies de partenariat et développement des organisations sportives dans les secteurs public et privé, Thèse de doctorat de sociologie, Université Marc Bloch, Strasbourg, 1997. Thèse reproduite et diffusée par les Presses Universitaires du Septentrion, Villeneuve d'Ascq, 1999.

Pichot L., "Partenariats sportifs des entreprises et logiques d'action des responsables de communication", in: revue STAPS, revue internationale des sciences du sport et de l'éducation physique, Grenoble, Ed. AFRAPS, 52, 2000, pp. 21-32.

Pichot L., «Les usages du sport par les entreprises : des stratégies entrepreneuriales aux stratégies des agents ", in: Regards sociologiques, Strasbourg, Ch. de Montlibert Ed., 20, 2000, pp. 53-65.

Pichot, L. et Tribou, G., «Sponsoring sportif: outil de communication externe et interne », in Bouchet P. et Pigeassou C. (dir.), Management du sport: actualités, développements et orientations de la recherche, Montpellier, Ed. AFRAPS, 2006, pp. 391-408.

Pouquet L., Le comportement des consommateurs d'articles de sport. Paris, Credoc. 1994.

Schaeffer P., Les machines à communiquer. Paris, Seuil. 1970.

Schlefen Albert E., Communicational Structure: Analysis of a Psychoterapy Transaction. Bloomington. Indiana University Press. 1973.

Schwebig P., Les communications de l'entreprise: au-delà de l'image. Mc Graw Hill. 1987.

Villette M., L'homme qui croyait au management. Paris, Seuil. 1988.

Williams R., Culture and society, Penguin. 1961.

Winkin Y. et al., La nouvelle communication. Paris, Seuil, coll. « Points ». 1981.

Winkin Y., Anthropologie de la communication, de la théorie au terrain. Paris. Seuil, coll. « Points », 2001

\section{Annexe méthodologique}

A partir de revues de presse, de sources d'information internes (journaux d'entreprise, discussions informelles avec les salariés), et surtout d'entretiens semi-directifs $(n=25)$ d'une durée d'une heure et demie, menés auprès de cadres décisionnaires des politiques de communication d'entreprises industrielles et de services $(n=15)$ positionnées sur des marchés régionaux, nationaux et/ou 
internationaux, notre recherche se focalise sur les politiques de communication utilisant le sport comme vecteur communicationnel en interne (circulation de l'information par les réseaux sportifs associatifs ou informels, formation du personnel dans le cadre de stages incentive) et en externe en termes de sponsoring sportif. Les cadres interrogés (directeurs de la communication, du marketing, chargés de sponsoring) participent, sinon à la définition de la politique, du moins à sa mise en œuvre. Les discours enregistrés des acteurs décisionnaires constituent la matière principale pour décrypter les logiques communicationnelles autour du sport. Les données exploitées s'inscrivent dans des travaux de recherche empirique personnels et ne sont pas le produit de rapports d'étude commandités, a fortiori répondant à un objectif politico-idéologique ou stratégique des directions d'entreprises. En ce sens, je ne suis pas lié contractuellement aux entreprises étudiées, je ne suis pas un satellite qui servirait de caution externe pour valider leurs pratiques. Pour pénétrer l'entreprise, je me suis présenté comme un enseignant dispensant des cours en communication d'entreprise exploitant le vecteur sportif, et non pas comme un chercheur en quête de données de terrain à des fins d'analyse scientifique. Cette approche qui dissimule l'objectif de l'entretien a pour intérêt de ne pas crisper les interlocuteurs se sentant évalués, et de ne pas recueillir un discours policé et convenu visant à « vendre » l'image désirée de l'entreprise. Il n'en demeure pas moins fragile que l'analyse repose essentiellement sur la parole d'un ou de deux interlocuteurs représentant l'entreprise et y occupant une position partiale et engagée. Les moyens de contrôle limitant ces biais consistent d'une part à montrer un niveau de connaissances minimales sur le thème abordé et, d'autre part, à croiser les informations de notre interlocuteur avec les propos tenus par les salariés. Si les croyances rapportées par les décideurs font partie de la réalité, cette dernière comprend aussi celles des salariés. A cet égard, la recherche présentée ici ne s'attache pas, à l'instar des modèles d'analyse positivistes, aux effets et à l'efficacité des actions de communication sur les collaborateurs. Seule une investigation menée auprès des salariés sur la réception des opérations de communication permettrait d'objectiver leur capacité performative, d'apprécier leurs effets sur l'affiliation sentimentale des acteurs sociaux à l'entreprise. 